\title{
Integrated System Design of Laser Seeker on Simulation, Test and Diagnosis
}

\author{
ZHAO Hanwu ${ }^{\mathrm{a}}$, YANG $\mathrm{Jia}^{\mathrm{b}}$ \\ Aviation Maintenance School for NCO, Air Force Engineering University, China \\ azhaohanwu@gmail.com, byeaperser@gmail.com
}

Keywords: laser seeker, simulation, test, fault diagnosis, integration

\begin{abstract}
To reduce product development period and make the best of information from different product phases, an integrated system is investigated in the paper. Based on requirement analysis of laser seeker development, test and fault diagnosis, the integrated system is built with each subsystem in detail, and an integrated information platform is proposed for data sharing and utilizing. Research results show that the system can boost the product development and improve reliability distinctly.
\end{abstract}

\section{Introduction}

Simulation is an important technical method in missile seeker research work. At the same time, product performance test is a key step in seeker's development, and fault diagnosis is a main requirement to ensure the realization of such performance. Existing seeker simulation, performance test or fault diagnosis platforms usually are standalone and focus on separate phase during the product R\&D or final application. Such situation results in that difficult contrast can be made during the entire product life cycles, thus the product development period is extended and the cost is also increased. Furthermore, for the application of the product, troubleshooting is hard and time-consuming since lack of according knowledge.

Recently a trend from standalone platform to integrated platform with simulation/test/fault diagnosis tasks wholly built-in arises [1, 2]. Such integration platform improves the reliability of product and facilitates the generation updating of product. Considering the requirements of a certain laser seeker during different phase of product, the paper proposes a new platform to accomplish laser seek simulation, test and fault diagnosis all in one.

\section{Design Principle}

The purpose of integrated system is to build an advanced and flexible system to accomplish multiple tasks including performance simulation in research, function test in development and fault diagnosis in product application. The principles include [3]:

Adopting modular hardware to achieve multiple functions. Common modules are used widely for low costs and convenience. Such architect also has rooms for further expansion.

Building integrated information platform. Such an information platform can merge multiple source information into it. An information platform also breaks the boundaries of information produced in different phases of the product R\&D and application.

Software design adopts modular principle also to realize the integration of performance simulation, comprehensive testing and fault diagnosis. Under the management and control of master software, each module inside the system can realize interacting and calling of other modules.

\section{System Design}

Integrated system aims to reduce the time on developing a new type of seeker and improve the efficiency and reliability of seeker usage and maintenance. The requirements of integrated system are summarized as following: digital simulation, which is accomplished by simulation computer and corresponding parts, is used for simulation. Performance test, which is accomplished by the 
seeker and turntable, is used to compute target-missile motion and turntable parameters, apply power to system and seeker and acquire the data produced by seeker. Fault diagnosis is used to give fault diagnosis report and maintenance indications.

Considering adaptability, maintainability and expansibility, the integrated system physically comprises main cabinet (including simulation computer, test computer and target simulation computer), turntable, the laser target simulator and data acquisition, and signal conditioning module et al. Fig. 1 shows the basic components of integrated system.

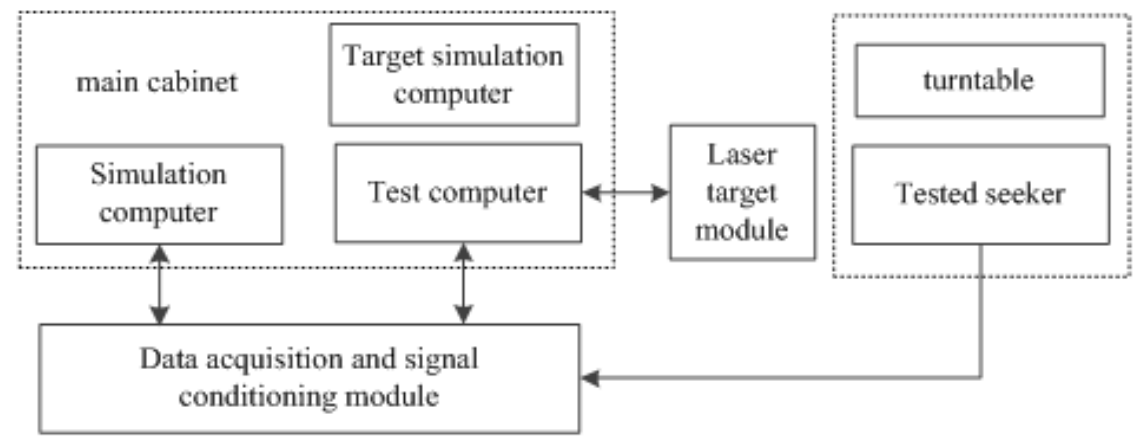

Fig.1 Integrated system component of laser seeker simulation, test and diagnosis

System Simulation Module. Simulation module aims to reduce the time of seeker verification and modification, boost the seeker development. Also, there will be plenty of data produced while simulation. These data can be utilized when testing or fault diagnosis.

System simulation module consists of initialization module, target-missile module, data recording module, 3-D viewing interface module and data acquisition module. The operating platform is based on LabVIEW and has graphical interface which is easy to use by operators among different product development phases.

System Test Module. Normally system test module has two tasks. Firstly, the tested seeker will be taken to have an open-loop test. System will measure the output of the seeker, and evaluate the track performance, stability and dynamic performance.

Secondly, the seeker will be taken to have a closed-loop test. Test module acquires the test data and passes them to the simulation module. That means the module can realize not only traditional testing task, but also the real-time analysis of simulation procedure. Based on LabVIEW RT real-time operating system and PXI hardware architecture, system test module can acquire the data from tested seeker and pass them to master computer by PXI-CAN board [4].

Fault Diagnosis Module. A fault diagnosis module is designed based on Fault Tree Analysis to realize fault positioning and diagnosis. It works by distinctly illustrating the fault diagnosis knowledge and experience from repair experts by Fault Tree [5]. After summing-up and abstracting, the Fault Tree is converted to diagnosis knowledge base and lead operators to repair step by step by graphical human machine interface. For example, the fault tree for target not caught by seeker is built as shown in Fig. 2.

Fault Tree Analysis consists of qualitative analysis and quantitative analysis. For fault tree building for seeker diagnosis, minimal cut sets by qualitative and quantitative analyses are investigated together [6]. The priority and reliability of minimum cut sets are computed. Then the diagnosis flow is created according to the order of importance of minimum cut sets in which operators can be lead to position the fault.

Data Processing Module. Integrated system is a data sharing platform, which establishes a formal and strict data format used in seeker simulation, testing and fault diagnosis. The system have specific interface to connect with modules handling different tasks during the product life cycle.

Software Module. System is based on Windows XP operating system and LabVIEW software. As shown in Fig. 3, applications inside the system software consist of six parts: system management, system simulation, system test, fault diagnosis, data processing and online monitoring. Fig. 3 shows the software architecture of the system.

Among the modules inside the software, system simulation, system test and fault diagnosis 
modules are important function applications. Under the control of master software, they take the responsibilities of corresponding functions. Data processing part is also an important part. It acts as an information system, which have all relative data for the seeker inside it. These data includes information from different seeker life cycles.

Besides these, system management module, data processing module, online monitoring module are also provided to accomplish corresponding tasks.

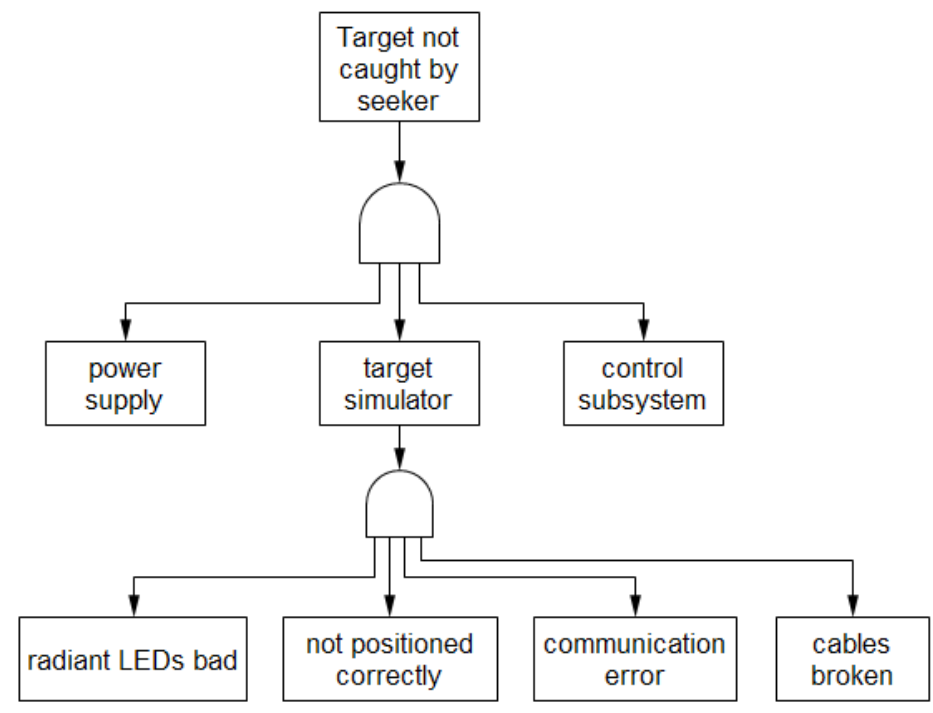

Fig.2 Fault tree example

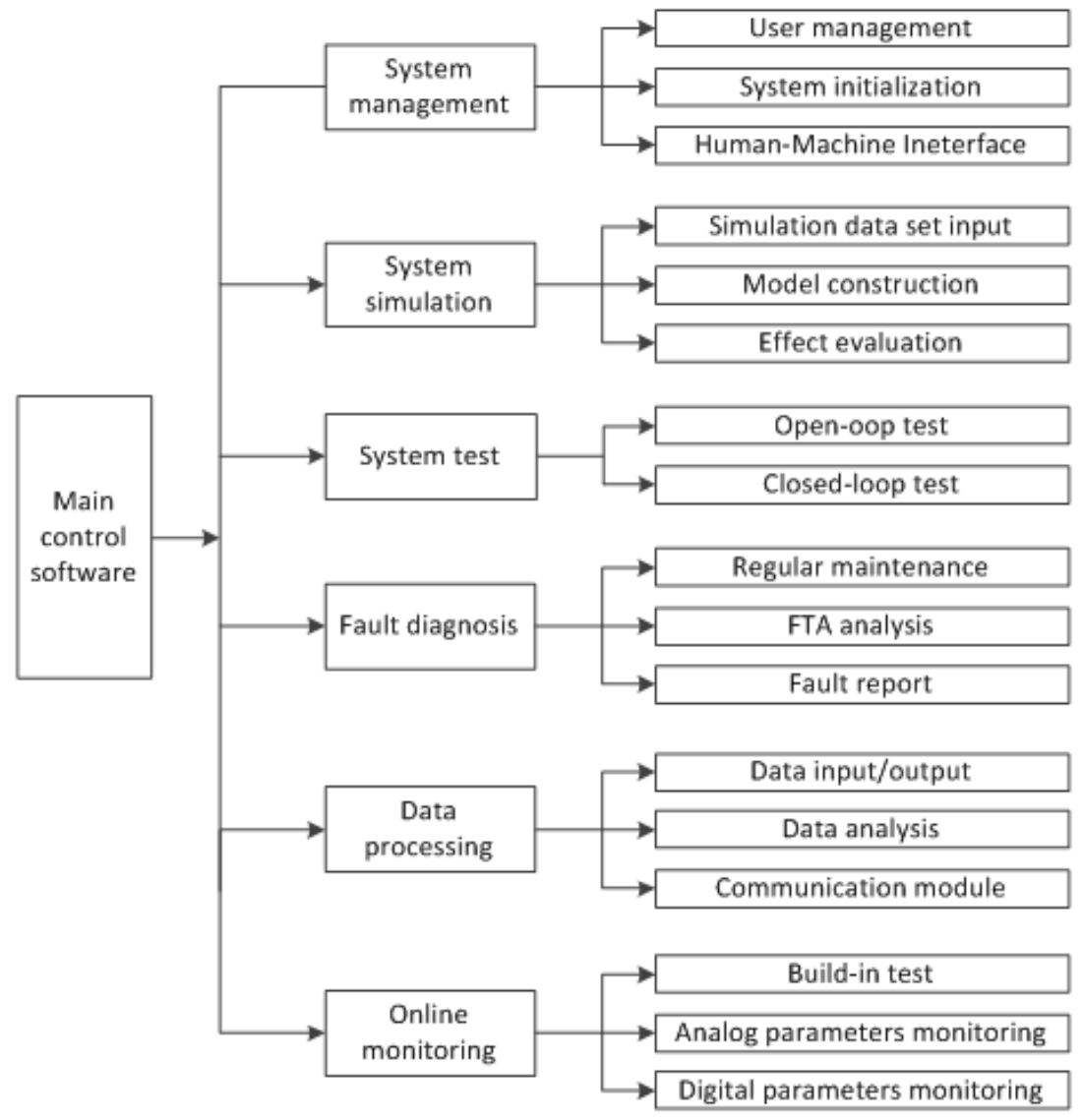

Fig.3 System main control software

\section{Summary}

An integrated system is investigated based on requirements analysis of laser seeker simulation, testing and fault diagnosis. Research results show that the system can meet the requirements of 
certain seeker simulation, testing and fault diagnosis and have the advantages of easy operating and good expansibility. Implement it into $R \& D$ of seeker product deeply will boost the product development and improve the reliability distinctly.

\section{References}

[1] LIU Guang-hui, ZHU Xiang, LV Tao, ZHOU Jun, WANG Mao, Design and investigation of simulation test integration for a seeker, Measurement \& Control Technology, Vol. 36(2017): 155-158.

[2] DU Jiang, LI Miao, WANG Tian-hui, Design of technology supporting system of radar seeker based on incorporated test and diagnosis, Computer Measurement \& Control, Vol. 22 (2014): 775-777.

[3] LIN Yuan, Research on integrated general test and fault diagnosis equipment, Tactical Missile Technology, (2009): 71-74.

[4] PU D L, SHANG X J, ZHANG W J, et al. An application of real-time operating system in high speed and high precision motion control systems, Proceedings of 3rd IEEE International Conference on Automation Science and Engineering, (2007): 997-1001.

[5] ZHU Da-qi, Principle and practice of electronic equipment fault diagnosis, Publishing House of Electronics Industry, Beijing, 2004, pp. 110-119.

[6] CAO Ming, LIU Ya-bin, Application of fault tree analysis to seeker fault diagnosis, Electronic Design Engineering, Vol. 24(2016): 118-120. 